\title{
Compression Schemes for In-body and On-body UWB Sensor Networks
}

\author{
Pål Anders Floor ${ }^{* \# 1}$, Ilangko Balasingham ${ }^{* \# 2}$, Tor A. Ramstad ${ }^{*}$, Eric Meurville ${ }^{\dagger}$, Michela Peisino ${ }^{\dagger}$ \\ *Interventional Center, Oslo University Hospital and Institute of Clinical Medicine, University of Oslo, \\ Oslo, Norway \\ 1andflo@rr-research.no ${ }^{2}$ ilangkob@medisin.uio.no \\ \# Dept. of Electronics and Telecommunications, Norwegian University of Science and Technology \\ Trondheim, Norway \\ ${ }^{\dagger}$ Swiss Federal Institute of Technology (EPFL), Lausanne, Switzerland
}

\begin{abstract}
Recently, there has been an increasing interest in applying wireless sensor networks for health status monitoring both on and within the human body. Low power consumption is crucial in such applications, especially for implanted devices. UWB transmission is one way of making low power transmission possible. A further reduction in power consumption can be achieved through efficient signal compression schemes. Since signals resulting from the measurements of physical phenomena are correlated compression prior to transmission can reduce the bit-rate an thus the power consumption significantly.

In this paper a compression schemes for a 2-node sensor network is studied. Differential pulse code modulation is applied for removal of temporal correlation and distributed quantization is used for exploitation of inter-sensor correlation. An example on optimization of encoders and decoders based on the statistical properties of an ECG signal is performed. Significant compression is shown to be achieved at low complexity.
\end{abstract}

\section{INTRODUCTION}

Wireless transmission of data from devices on and within the human body is a challenge, especially when it comes to implants which must be very small, reliable and have a very low power consumption.

A study on electromagnetic wave propagation in the human body shows that high attenuation is a big obstacle [1]. This makes low power transmission a big challenge. However, the problem can be reduced because power can be traded for bandwidth as long as the channel bandwidth is much larger than the bandwidth of the source to be transmitted [2]. By considering Ultra wideband (UWB) communication, then transmission power can in principle be kept relatively low. The question is if there are possibilities to further reduce power consumption. One possibility is to apply compression. Compression schemes applied in medical sensor networks should result in a low rate per source sample per sensor while keeping the encoders (sensors) as simple as possible. The decoder (fusion center) can, however, be more complex. The objective of this paper is to investigate potential compression schemes for medical sensor networks.

Signals resulting from the measurement of physical phenomena will be correlated in time. Correlation is redundant and can, in principle, be removed from relevant signals at the transmitter and added at the receiver without any loss of fidelity. In applications where power is a scarce resource it is important to remove as much redundancy as possible prior to transmission with the lowest possible computational resources so that the waste of power is kept at an absolute minimum. A potential scheme for the removal of temporal correlation is differential pulse code modulation (DPCM), which is known to provide significant compression at low complexity [3].

Exploiting correlation between different sensors is another possibility for lowering signal rates per sensor. A distributed scheme is most beneficial, especially for implants, since it does not require the implementation of receivers in the sensors. It is also hard to make implants cooperate. Distributed quantization (DQ) [4] is a potential scheme. In DQ the quantizers for several sensors are co-optimized taking known inter-sensor correlation into account. Significant gains can be achieved if correlation is high. DQ can be used in combination with DPCM to exploit both temporal and inter-sensor correlation simultaneously.

In this paper DPCM compression and DQ are explored both separately and in combination for a simple sensor network consisting of two sensors and one joint receiver. An example on optimization of encoders and decoders based on the statistical properties of an ECG signal is given. The proposed scheme provides significant compression at low complexity.

In Section II and III the theoretical principles behind DPCM and DQ are described. In Section IV DPCM and DQ is optimized for an ECG signal. Noise, attenuation and nonstationarity will also be briefly addressed. In Section $\mathrm{V}$ a discussion is given and conclusions are drawn.

\section{DPCM COMPRESSION}

Fig. 1 shows a closed loop DPCM encoder and decoder. In the encoder (Fig. 1(a)) compression is achieved by exploiting correlation in the input signal $x[n]$ to predict the current sample based on $P$ previously measured samples using a Pth- 


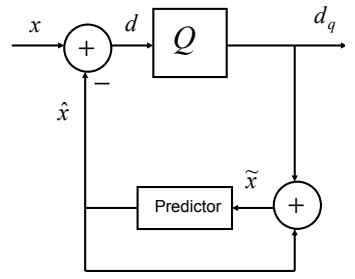

(a)

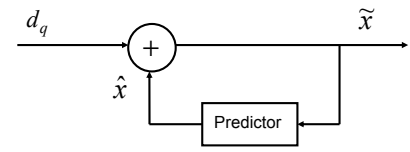

(b)
Fig. 1. DPCM scheme. 1(a) Encoder. 1(b) Decoder.

order predictor (P-order FIR filter)

$$
\hat{x}[n]=c_{1} \tilde{x}[n-1]+c_{2} \tilde{x}[n-2]+\cdots+c_{P} \tilde{x}[n-P],
$$

where $c_{i}$ are the prediction filter coefficients which must be optimized for the relevant signals statistics and $n$ is the time index. In DPCM the quantized prediction error

$$
d_{q}[n]=Q(d[n])=Q(x[n]-\hat{x}[n]),
$$

i.e. the quantized difference between the original samples and the predicted ones, is communicated. In the decoder (Fig. 1(b)) the original signal will be recovered as

$$
\tilde{x}[n]=d_{q}[n]+\hat{x}[n],
$$

If the signal is highly correlated and the optimal predictor is chosen, the error signal will usually be small. DPCM might perform poorly if the signal contains many non-periodic impulses however.

Given a certain maximum allowed distortion the necessary number of bits per sample will be reduced by quantizing the error signal instead of the original since the prediction error has a lower dynamical range.

\section{A. Determination of optimal prediction filter coefficients}

The optimal prediction filter coefficients are found by solving the Yule-Walker equations [5, p. 343]

$$
\mathbf{R}_{x} \mathbf{c}=\sigma_{d}^{2} \mathbf{l},
$$

where $\mathbf{R}_{x}$ is the $(P+1) \times(P+1)$ autocorrelation matrix for the relevant signal $x, \mathbf{c}=\left[\begin{array}{llll}1 & c_{1} & \cdots & c_{P}\end{array}\right]^{T}$ are the prediction filter coefficients, $\sigma_{d}^{2}$ is the variance of the error signal $d[n]$ and $\mathbf{l}=\left[\begin{array}{llll}1 & 0 & \cdots & 0\end{array}\right]^{T}$. Note that the coefficients which belong to a solution of (4) give the estimate representation $\hat{x}$ of $x$. In DPCM the prediction error representation is wanted, and so the sign of all the coefficients must be inverted to get the correct result $\left(a_{i}=-c_{i}\right)$. If the statistics of the random process we want to predict is stationary, fixed coefficients can be applied. If the statistics is changing, however, the coefficients should be adapted. The number of coefficients needed can be determined from the resulting prediction error in any case. If $\sigma_{d}^{2}$ is not significantly decreased by adding more coefficients, the correct number has been found. Since the prediction filter is involved in a feedback loop, one must ensure stability of the whole loop, named prediction error filter.
In most cases the autocorrelation matrix is unknown and must be estimated from the relevant data. Assume that $N_{s}$ samples from a stationary ergodic process $x$ is available. An unbiased estimate of the correlation matrix is given by

$$
\hat{\mathbf{R}}_{x}=\frac{1}{N_{s}-M+1} \mathbf{X}^{T} \mathbf{X}
$$

where [5, pp.307-310]

$$
\mathbf{X}=\left[\begin{array}{cccc}
x[M-1] & x[M-2] & \cdots & x[0] \\
x[M] & x[M-1] & \cdots & x[1] \\
\vdots & \vdots & \ddots & \vdots \\
x\left[N_{s}-1\right] & x\left[N_{s}-2\right] & \cdots & x\left[N_{s}-M\right]
\end{array}\right]
$$

and $M=P+1$.

\section{Distributed QuAntiZATION}

Distributed quantization (DQ) can be deployed to lower the number of bits required per sample, or the necessary transmission power for a given target fidelity when several sensors are measuring one or several highly correlated physical processes. In this paper the simple network in Fig. 2 will be explored. We want to determine the best possible mappings $f_{1}$,

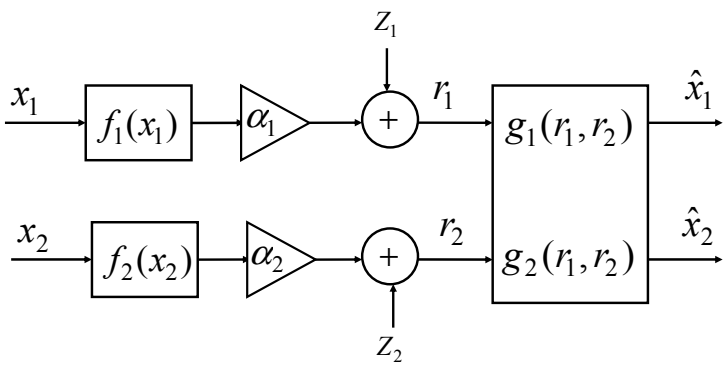

Fig. 2. 2-node sensor network.

$f_{2}, g_{1}$ and $g_{2}$. For the special case where two equal variance Gaussian memoryless sources are communicated on Gaussian channels with unit attenuation $\left(\alpha_{1}=\alpha_{2}=1\right)$ the optimal performance is known [4]. This special case can be used to illustrate the concept and to indicate when DQ is applicable. For any possible choice of encoders and decoders $\left(f_{1}, f_{2}, g_{1}\right.$ and $g_{2}$ ) the performance will be limited by [4]

$$
S D R=\left(\left(1+\frac{P}{\sigma_{n}^{2}}\right)^{-2}\left(1-\rho_{x}^{2}\right)+\rho_{x}^{2}\left(1+\frac{P}{\sigma_{n}^{2}}\right)^{-4}\right)^{-\frac{1}{2}}
$$

where SDR is the received signal-to-distortion ratio, $P$ is the maximum allowed power per channel, $\sigma_{n}^{2}$ is the noise variance and $\rho_{x}$ is the correlation coefficient for the two sources. We call this the ideal case. Fig. 3 shows the optimal performance for several values of $\rho_{x}$ as well as a practical 5-bit scalar DQ scheme optimized at $\approx 20 \mathrm{~dB}$ channel SNR using the procedure in [4]. Some important conclusions can be drawn from these results. One can observe that even in the ideal 


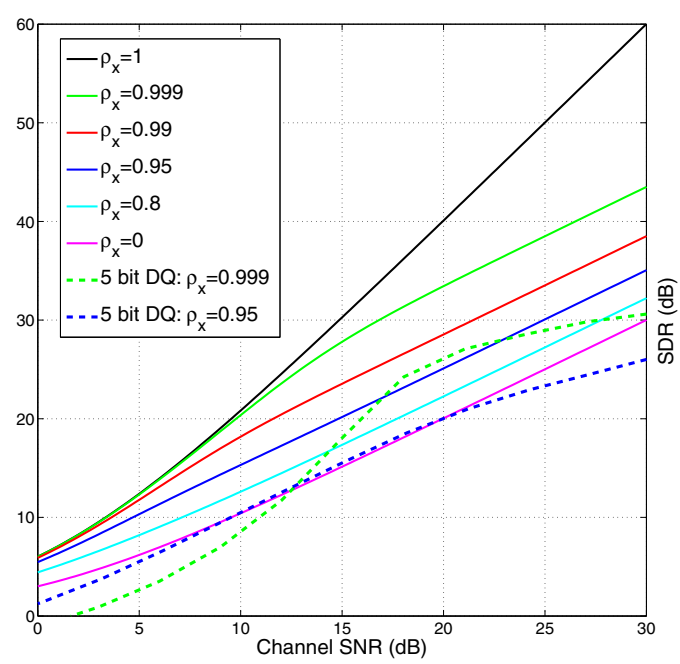

Fig. 3. Performance of 2-node GSN. The whole lines represents the ultimate performance while the dashed represents 5 bit DQ.

case $\rho_{x}$ must be relatively close to one for the gain ${ }^{1}$ to be significant. Notice specifically that the gain is increasing faster with $\rho_{x}$ above 0.8 than below. For a practically realizable scheme like DQ one must expect $\rho_{x}$ to be even larger to achieve significant gain. For 5 bit DQ there is little or no gain before $\rho_{x}>0.95$. For $\rho_{x}=0.999$ the gain is significant even for 5 bit DQ.

Therefore, with 5 bit scalar quantization, DQ can be potentially applied when $\rho_{x}>0.95$, while "ordinary" quantization in each sensor will do just as well for lower values of $\rho_{x}$.

Why do we get the behavior in Fig. 3? The answer lies in geometry and will make it possible to do reasoning for more general cases than what is considered here. In Fig. 4 the encoders $f_{1}$ and $f_{2}$ (which now represents the quantizer indices) for 5-bit DQ are shown. When $0<\rho_{x}<0.95$ the

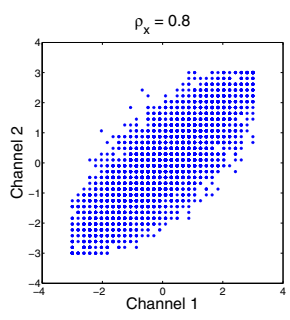

(a)

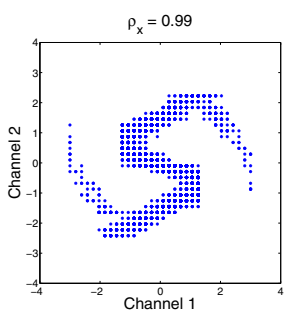

(b)
Fig. 4. Index configuration in the channel space for 5 bit DQ. 4(a) $\rho_{x}=0.8$. 4(b) $\rho_{x}=0.99$.

indices will lie in an elliptical region like in Fig. 4(a) reflecting the pdf of the input signal vectors. The ellipse must lie within a region determined by the channel power constraint. As the

\footnotetext{
${ }^{1}$ Note that by gain we here mean the increase in SDR for a given SNR compared to the $\rho_{x}=0$ case (the magenta line).
}

correlation increases the elliptical region will "narrow". When $\rho_{x}$ increase above $\approx 0.95$ the ellipse becomes so narrow that much of the channel space is empty. This empty region can be exploited for noise reduction by "filling" the channel space as properly as possible through a nonlinear mapping (nonlinear $f_{1}$ and $f_{2}$, as in Fig. 4(b)). This filling must be done without significantly increasing the probability for threshold effects. Threshold effects result in large decoding errors and occur when points on different "folds" of the given structure (like the one shown in Fig. 4(b)) are interchanged. The result of threshold effects can be observed in Fig. 3, which is the reason why the scheme optimized for $\rho_{x}=0.999$ deteriorates relatively fast below the optimal cannel SNR. Trying to fill the channel space through nonlinear mappings when $\rho<0.95$ will either violate the power constraint or increase the probability for the threshold effect significantly. For the cases where nonlinear mappings can be applied, quantizer indices can be re-used for several quantization intervals ${ }^{2}$, and so, for a given number of indices (a fixed rate), it is possible to increase the resolution. For example, using 5 bit DQ when $\rho_{x}=0.99$ the signal of one source can be quantized into 49 different levels even though only 32 indices are available. This effect will be more pronounced when the correlation is larger and when quantizers for more than 2 sensors are co-optimized.

\section{DPCM AND DQ APPLIED ON ECG SIGNALS}

In this section DPCM and DQ will be applied for compression of ECG signals. Furthermore the effect that heart rate variability, arrythmia, attenuation and noise has on the suggested schemes will be discussed.

When diagnosing the heart it is the shape of the ECG signal and the relation between the different peaks (the QRS complex) that matters. We will therefore use the visual quality in the reconstruction as a measure of performance for the suggested schemes.

All examples in this section will be done on $250 \mathrm{~Hz}$ ECG data downloaded from the MIT-BIH Arrythmia Database. We illustrate the performance on a relatively stationary ECG record. Non-stationarity will be addressed in Section IV-C.

\section{A. Compressing ECG signals with DPCM}

Similar work has been done before in e.g. [6], [7]. We do our own optimization here to illustrate the significant compression achieved by this scheme, and to combine it with DQ in Section IV-B. The DPCM encoders and decoders are optimized for a vector of 100000 samples. 1st-, 2nd- and 3rd order predictors are tested. Forming the matrix in (6) inserting $N_{s}=100000, M=P+1=2$ and performing the calculation in (5), gives the wanted matrices. Inserting these matrices in the Yule-Walker equations (4), solving for the coefficient vectors and further inverting the signs give the wanted coefficients $a_{i}$. Fig. 5(b) depicts the 2nd order predictor and Fig. 5(a) shows the original ECG signal and

\footnotetext{
${ }^{2}$ Reuse of indices leads to ambiguities at the encoders, but since each pair of indices is unique, the ambiguity will be resolved through observation of both indices at the receiver.
} 
the error signals from the three predictors. It is clear that

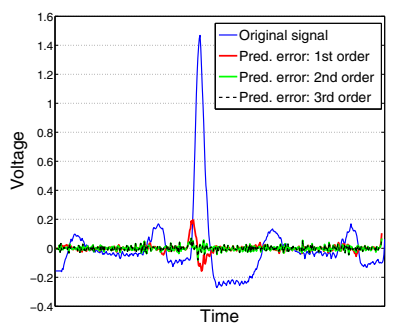

(a)

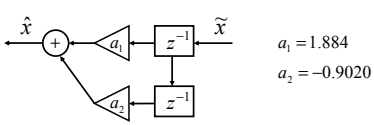

(b)
Fig. 5. Prediction. 5(a) Comparison between the original ECG signal and the prediction error signal for 1st-, 2nd- and 3rd order DPCM. 5(b) 2nd order predictor constructed using ECG data.

the dynamical range of the signals are significantly reduced through DPCM compression. The ratio between the average power of the original signal and the average power of the compressed signal is 52, 280 and 283 for 1 st, 2nd and 3rd order predictors, respectively. It is apparent that a 2nd order predictor is favorable for this specific case. There is a lot to gain in going from a 1 st to a 2 nd order predictor, while going from a 2 nd to a 3 rd order predictor gives a negligible gain. This will be the case for most ECG records.

To illustrate the benefits of DPCM, its performance is compared to direct quantization of the ECG signal. Fig. 6(b) and 6(c) show the reconstruction when using DPCM and direct quantization respectively for 3 bits. The difference between the original signal and the DPCM coded signal is small. Direct quantization results in severe distortion. One must use $\approx 7$ bit direct quantization to achieve the same quality as 3 bits DPCM.

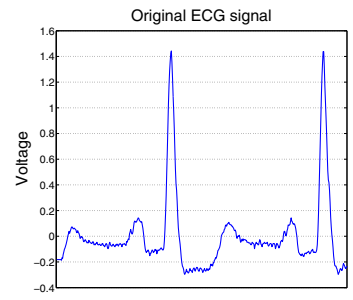

(a)

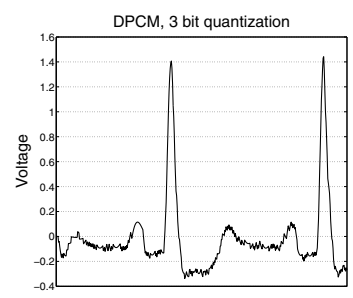

(c)

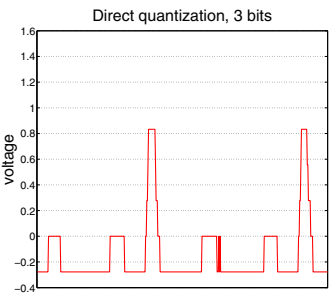

(b)

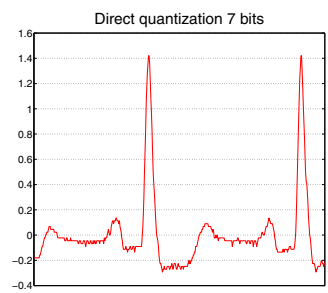

(d)
Fig. 6. ECG compression. 6(a) Original ECG signal. 6(b) 3 bit direct quantization. 6(c) 3 bit DPCM. 6(d) 7 bit direct quantization.

\section{B. DPCM and DQ used in combination}

Consider two highly correlated sensors (i.e. $\rho_{x}=0.9999$ ) both measuring an ECG signal. We will show results for both direct DQ and DQ applied to the DPCM error signals from the two sensors. The DQ need to be optimized for the relevant signal statistics. We choose to fit a Generalized Gaussian Distribution (GGD) to the relevant data by applying the estimation procedure described in [8, pp. 47-48]. The results show that the DPCM error signal is relatively close to Laplacian and fits well to a GGD estimate. The ECG distribution is nonsymmetric and has a double peak (due to its highly nonsymmetric oscillation around 0 Volt), and therefore does not fit perfectly to a GGD. The best GGD estimate is, however, close to Laplacian. We therefore choose to optimize the DQ for a Laplacian distribution for both cases using the procedure developed in [4]. Notice that when constructing DQ for the DPCM error signal, it is crucial that the centroids in the encoder and decoder are of the same value, or else severe distortion will result from the DPCM decoder. This excludes the use of MMSE decoding in the DQ. For the same reason is is also assumed that the channel noise is so low that errors do not occur (see Section IV-D).

Fig. 7(b) shows the result of direct DQ using 3 bits per sensor and Figure 7(d) shows the result of combined DPCMDQ using 2 bits per sensor. A comparison with Figure 6(b) show that DQ gives a significantly better resolution than ordinary quantization for the same number of bits per sensor (the signal has been clipped at $\approx 0.8$ volts in order to get a better resolution of the whole QRS complex). The combined DPCM-DQ scheme has the best performance and gives a reconstruction almost indistinguishable from the original using only 2 bit quantization per sensor.

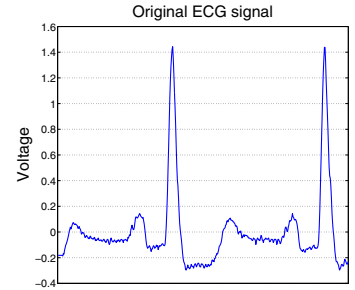

(a)

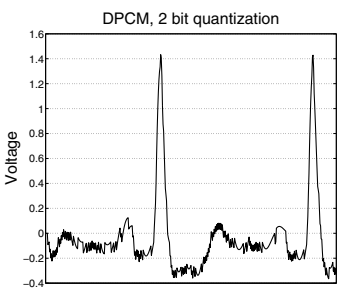

(c)

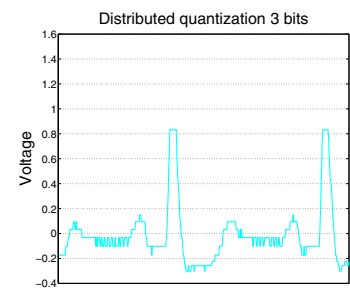

(b)

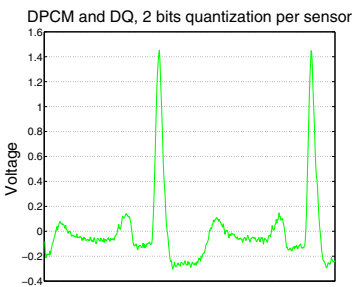

(d)
Fig. 7. ECG compression. 7(a) Original ECG signal. 7(b) DQ 3 bits per sensor. 7(c) 2 bit DPCM. 7(d) DPCM + DQ, 2 bits per sensor. 


\section{Non-stationarity}

The statistics of the ECG signal change over time both due to normal variations (e.g. the increase of pulse rate) and due to arrythmia and heart failure. This will affect the performance of DPCM. When the statistics change the predictor optimized for a stationary ECG signal will perform worse leading to an increase in the error signals dynamical range which might lead to overload errors in the quantizer.

Changing pulse rate can be accounted for by using some sort of peak detection. Several algorithms for detecting Rcomplexes (the main peak of the ECG signal) exists like the one described in [9]. To achieve a relatively constant dynamical range in the error signal for all pulse rates one can adapt the predictor coefficients by re-solving (4) every time the rate of the R-complexes changes. The Levinson recursion [5, pp. 422-430] is a fast way of solving a linear system of $n$ equations in $O\left(n^{2}\right)$ arithmetic operations. For a 2 nd order predictor, this will be quite efficient and done with $\approx 4$ arithmetic operations. One must also re-estimate $R_{x}$ where the complexity depend on the number of samples used. The number of samples needed will probably be a tradeoff between complexity and accuracy and is a subject for future research. Also note that if a good feedback channel is available the predictor in the DPCM encoder can be moved to the receiver [2] implying that the sensors will be of very low complexity even though an adaptive scheme is used, since all the processing can be done at the fusion center. This will, however, require implementation of receivers in the sensors.

The statistics also change when the ECG become abnormal (arrythmia and heart failure) leading to a poorer prediction. Some redundancy removal will still be present, but the error signals dynamical range might increase dramatically. Adaptation of the coefficients is a possibility to avoid this effect. Alternatively, the increase in the error signal may be used as a warning sign that something critical is about to happen. But then a method must be found that makes it possible to differentiate between normal variations and arrythmia.

\section{Transmission}

As mentioned earlier, the attenuation of signals from implants can be quite severe. This combined with the desired low power consumption require efficient and simple transmission schemes. With access to ultra-wide-band (UWB) transmission, a viable transmission scheme is pulse position modulation (PPM). The principle is to transmit a pulse in one out of $N=2^{b}$ positions per frame, implying that $b$ bits are transmitted per frame. Transparent transmission is obtained when all positions belong to a Nyquist channel and thus can be detected optimally using the correct correlator, while at the same time the received pulse energy is sufficiently larger than the power spectral density of the noise. The necessary pulse energy increases only marginally when the number of positions is increased, which implies that the energy per bit is almost inversely proportional to $b$. Notice, however, that the required bandwidth is proportional to $N$.
Assuming that the DPCM coder uses $b_{e}$ bits per sample and the sampling frequency is $f_{s}$, then the required channel bandwidth is $B=0.5 f_{s} b_{e} 2^{b} / b$. The required energy per sample can be calculated based on the transmission attenuation and analysis given in [10, ch.8] when requiring a certain bit error rate.

\section{Discussion AND CONCLUSIONS}

In this paper the use of differential pulse coded modulation (DPCM) and distributed quantization (DQ) for compression in medical UWB sensor networks has been addressed.

For highly correlated signals DPCM will give significant compression. DQ can be used to compress signals from two or more sensors in the case of high inter-sensor correlation. If correlation is not high there may be little to gain from DQ and the sensors can just as well be constructed separately.

Examples on compression of ECG signals using the suggested schemes showed promising results. I.e. one can reduce the number of bits per sensor necessary to a achieve a certain fidelity significantly compared to direct quantization in each sensor at relatively little cost in terms of computational complexity.

Future work will include optimization of the suggested schemes to other relevant signals as well as the channel models developed in [1], [11].

\section{ACKNOWLEDGMENT}

This work is part of the ULTRASPONDER project founded by the EU, FP7, STREP under the contract number 224009 and the MELODY project founded by the research council of Norway under the contract number $187857 / \mathrm{S} 10$.

\section{REFERENCES}

[1] A. Khalegi, R. Chávez-Santiago, X. Liang, I. Balasingham, V. Leung, and T. A. Ramstad, "On ultra wideband channel modeling for in-body communications," in International symposium on wireless pervasive computing. IEEE, May 2010.

[2] T. A. Ramstad, "Simple and reliable image communication based on dpcm and multiple refinements through feedback," in 3rd International Symposium on Communications, Control and Signal Processing. IEEE, Mar. 2008.

[3] B. Beferull-Lozano, R. L. Konsbruck, and M. Vetterli, "Rate-distortion problem for physics based distributed sensing," in International Conference on Information Processing in Sensor Networks. IEEE, Apr. 2004.

[4] N. Wernersson, J. Karlsson, and M. Skoglund, "Distributed quantization over noisy channels," IEEE Trans. Commun., vol. 57, no. 6, pp. 1693 1700, June 2009.

[5] C. Therrien, Discrete Random Signals and Statistical Signal Processing. Prentice Hall, 1992.

[6] S. M. Jalaleddine, C. G. Hutchens, R. D. Strattan, and W. A. Coberly, "Ecg data compression techniques - a unified approach," IEEE Trans. Biomedical Eng., vol. 37, no. 4, pp. 329-342, Apr. 1990.

[7] C. S. G. Perera, Multiple Sensor Data Analysis, Fusion, and Communication for ULTRASPONDER. Master thesis, NTNU, 2009.

[8] J. M. Lervik, "Subband image communication over digital transparent and analog waveform channels," Ph.D. dissertation, NTNU, 1996.

[9] J. Pan and W. J. Tompkins, "A real-time qrs detection algorithm," IEEE Trans. Biomedical Eng., vol. 32, pp. 230-236, Mar. 1985.

[10] J. M. Wozencraft and I. M. Jacobs, Principles of Communication Engineering. New York: John Wiley \& Sons, Inc, 1965.

[11] K. Y. Yazdandoost and K. Sayrafian-Pour, "Channel model for body area network (ban).” IEEE 15-08-0780-07-0006, Mar. 2009. 